\title{
Innovative method to produce high-purity Graphitic Carbon Nanospheres.
}

\begin{abstract}
The present paper describes a method for producing graphitic carbon nanospheres (GCNSs) by chemical vapour deposition (CVD), which includes supplying carbon atoms to a catalyser for forming carbon nanoparticles in a controlled manner, in which the amount of carbon supply is controlled in relation to the time and temperature parameters. In this method short carbon fibres are used as supporting substrate for the mass formation of pure graphitic carbon nanoparticles in a fluidized bed reactor chemical vapour deposition (FBCVD). The synthesis of graphitic carbon nanoparticles (GCNPs) is carried out by the catalytic decomposition of a carbon source over a substrate deposited with catalyst in the fixed bed reactor. The chemical composition of nanoparticles was measured by energy dispersive X-ray spectrometry (EDX) in a scanning electron microscope (SEM). SEM, High Resolution Transmission Electron Microscope (HRTEM), thermal gravimetric analyser (TGA), and X-ray diffraction (XRD) were used for characterization of the catalyst and the products. This study indicates that GCNP is successfully grown at $600^{\circ} \mathrm{C}$ in ambient pressure for $1.8 \% \mathrm{Fe}$ on carbon fiber, 8 minutes deposition time, and 15L/min acetylene flow rate. Graphitic carbon nanospheres have been produced in large amount of lattice images of various fullerenic carbons and aciniform of nanoparticles, which are seldom reported in the literature.
\end{abstract}

Keyword: Mass production; Graphitic structure; Carbon nanosphere; Iron catalyst; Low temperature. 